\title{
EDITORIAL
}

\section{Medically Unexplained Physical Symptoms}

\author{
Jeffrey L. Jackson, MD, MPH', Susan George, $M D^{2}$, and Sherri Hinchey, $M D^{2}$ \\ ${ }^{1}$ Uniformed Services University, Bethesda, MD, USA; ${ }^{2}$ Walter Reed Army Medical Center, Washington, DC, USA.
}

$\mathrm{J}$ Gen Intern Med 24(4):540-2

DOI: $10.1007 / \mathrm{s} 11606-009-0932-\mathrm{x}$

() Society of General Internal Medicine 2009

$\mathrm{P}$ hysical symptoms are the most common reason patients seek medical attention. Among those presenting with symptoms, up to a third remain unexplained, even after thorough investigation ${ }^{1}$. Fortunately, most patients with unexplained symptoms improve and prospective studies show that the rate of resolution is similar to patients with explained symptoms ${ }^{2}$. However, there is a subgroup with persistent unexplained symptoms. There are numerous colorful descriptions of such patients, such as "difficult," "frustrating" or "heartsink,"3 with heartsink referring to the feeling clinicians have when they see a particular name on their schedule. While many patients have unexplained symptoms, the term "medically unexplained symptoms" has taken on a life of its own. Studies investigating the characteristics of "difficult" patient encounters reveal that both patient and physician characteristics play a role. ${ }^{4}$ These patients have multiple, persistent, unexplained physical symptoms. Their symptoms are more severe; they have poor functioning and tend not to improve over time. There is some evidence that less experienced clinicians and those with poorer psychosocial orientation experience a greater percentage of encounters as difficult. Both parties in these encounters emerge unsatisfied and short-term outcomes are poor.

There are numerous reasons why clinicians feel this way, including discomfort with diagnostic uncertainty, inadequate training in managing these patients and frustration at not being able to help their patients improve. This frustration at lack of definite diagnosis, for both patients and providers, has led to creation of a number of symptom syndromes, such as chronic fatigue syndrome, irritable bowel syndrome, fibromyalgia syndrome, chronic pelvic pain syndrome. Aaron studied these symptom syndromes and demonstrated a great deal of overlap between them ${ }^{5}$. All tend to have numerous, persistent unexplained symptoms with poor functioning and respond poorly to medical intervention. There is also evidence of a physiological cause for some of the problems. Functional MRI studies have found increased activity in areas of the brain that attend to symptoms, a reflection of potential hypervigilance ${ }^{6}$.

Everyone, upon reflection and self-examination, can find a litany of symptoms at any given moment. Most of us ignore the daily aches and pains of life. Patients with symptom syn-

The opinions reflected in this editorial are those of the authors and should not be construed, in any manner to reflect those of the US Army or the Department of Defense.

Published Online March 3, 2009 dromes may be biologically vigilant of these issues. Other studies have found that patients with these symptom syndromes also experience stimuli as painful at lower thresholds. ${ }^{7}$ Patients with irritable bowel syndrome report pain at lower rectal balloon inflation pressures, and those with fibromyalgia experience skin stimuli to be painful at lower pressures. It is interesting to speculate on common underlying biological mechanisms that then manifest themselves differently in particular individuals. Patients with more gastrointestinal predominant symptoms may be labeled as having irritable bowel syndrome, others with more myalgias receive fibromyalgia as a diagnosis, yet others with predominant fatigue are told they have chronic fatigue syndrome. While these patients have been given labels that distinguish their symptoms, they may have common underlying biological processes.

Most patients with persistent symptoms do no meet criteria for any of the DSM-IV somatization disorders. This has prompted the creation of a new diagnosis, multisomatoform disorder $^{8}$. To have multisomatoform disorder, patients must have three or more unexplained symptoms that have persisted 2 years. About $8 \%$ of patients in primary care meet these criteria $^{9}$. The majority have comorbid depression, which may explain some of the limited efficacy for antidepressants in patients with symptom syndromes. Nearly all patients with medically unexplained symptoms, particularly those who evoke a visceral response from clinicians will have multisomatoform disorder. Despite these efforts to refine the nomenclature regarding these patients, questions still remain. Are some labels more acceptable to patients or clinicians than others? Would one particular label be more useful in fostering patient-clinician dialogue? It seems clinicians perceive better acceptance among their patients for irritable bowel syndrome or fibromyalgia than multisomatoform disorder or other psychiatric diagnosis.

Studies have shown that communication in "difficult" encounters is generally poor. Part of the problem may be that patients with medically unexplained symptoms are a heterogeneous group. Some are seeking a diagnosis, others confirmation of their own models of illness which may vary from largely biological ("I have arthritis") to psychosocial ("I have stress in my life"). Identifying the specific patient's expectations for the consultation and belief about their illness can be difficult. Patients commonly have a "hidden agenda" that they may not readily share with their clinician. Salmon's study in this issue of JGIM suggests that patient desires may be inferred from the content of their talk. While this may be a starting point, other studies of patient-doctor communication suggest that patients are passive; it is not always obvious what they are seeking from medical encounters, even when directly asked by their clinician. ${ }^{10}$ What is needed is an easy method for clinicians to readily identify what patients are seeking from 
their visit. The dissonance between clinician perspectives of patient desires and actual patient desires contributes to encounter difficulty.

Clinicians are often hesitant to engage in psychosocial talk, because they feel patients are reluctant to explore this dimension of their symptom experience. In the absence of open dialogue, clinicians make assumptions about what patients' desire. One assumption is that patients are seeking definitive diagnosis and treatment. This resonates with primary care clinician's self-image as diagnosticians and healers. Clinicians live with uncertainty, but lurking in every clinician's mind is the possibility that they may have missed something. Many rare diagnoses have common and vague symptoms. When can diagnostic testing be deferred? In Salmon's study, patients provided cues in their conversation about their unstated and implicit desires. ${ }^{11}$ Patients who reported seeking emotional support engaged in more talk about psychosocial aspects of their problem. Patients seeking explanations suggested more physical explanations, though in either case, patients were rarely explicit in communicating their desires. It is unfortunate that clinicians and patients are left to swim in a sea of uncertain communication.

Peter's article in this issue of JGIM provides some clarification about why patients are hesitant to be explicit ${ }^{12}$. Patients have complex models of their own symptom experience and are reluctant to have this model oversimplified. While they are willing to admit a psychosocial component to their illness, they fear that clinicians will exclusively focus on this dimension, both for the current symptom and for future symptoms. Clinicians can overcome this barrier by working with the patient to acknowledge and address the psychosocial aspects of the patient experience, while also keeping this in balance with other aspects of patient care.

Several teaching aids have been devised to assist clinicians in dealing with psychosocial aspects of medicine, including "NURSE" and "BATHE" and "reattribution." Reattribution is a structured consultation delivered by general practitioners to provide psychological explanations to patients with multiple unexplained symptoms. All of these techniques attempt to improve physician communication with patients regarding the meaning and experience of their symptoms, with the goal of shifting focus of care from cure to coping. When rigorously studied, they improve patient satisfaction and clinician comfort in managing patients with medically unexplained symptoms. ${ }^{13}$ Uniformly, they have little or only modest impact on various measures of symptom related health. ${ }^{14}$

What is effective for treating symptoms in these patients? Pharmacological management of patients with medically unexplained symptoms centers on symptomatic treatment and anti-depressants. Even though numerous studies have suggested that patients with medically unexplained symptoms are not always seeking a prescription, most receive one. The effectiveness of symptomatic treatment has rarely been studied, but most studies suggest only minimal benefit. ${ }^{15}$ Anti-depressants have been used for numerous symptoms syndromes ${ }^{16}$. Most have minimal to modest effect on the underlying symptom and most studies are relatively short in duration, it is uncertain whether this efficacy will persist over time. Since many patients with symptom syndromes develop depression, monitoring and treating depression is appropriate. Unfortunately, treating depression improves depressive symptoms; effects on concomitant phys- ical symptoms have been disappointing. On the other hand, cognitive behavior therapy (CBT) has been demonstrated to be effective for numerous symptoms ${ }^{17}$, and many common management strategies have components of CBT built in. In order to move to this level of management, patients have to accept this shift in treatment goal from diagnosis and cure to amelioration and coping.

In the absence of making a diagnosis and prescribing definitive treatment, managing patients with medically unexplained symptoms becomes a task of negotiating clinician and patient expectations. Effective communication is paramount, but can be more difficult in these patient interactions. Patient centered strategies offer a framework for approaching this communication challenge and with practice can reduce clinician discomfort with such patients and provide a management strategy. These strategies seek to build a dialogue between patients and clinicians regarding their experience of symptoms, acknowledging and addressing both physical and psychological aspects. Exploring the psychosocial domain can be enriching and satisfying for both clinicians and patients. Over time, a balance needs to be struck between attributing new symptoms to psychosocial causes and exploring potential biologic ones. In practice, performing a careful history and physical examination and limiting additional diagnostic tests to those indicated by history and physical findings can reassure patients that their complaints will receive careful attention and not be dismissed. Patient-doctor communication is a vital cornerstone for any successful patient interaction, particularly among patients with medically unexplained symptoms. In an atmosphere of mutual trust and respect, "difficult" patient encounters can be transformed to richly satisfying ones for both patients and clinicians.

Corresponding Author: Jeffrey L. Jackson, MD, MPH; Uniformed Services University, Bethesda, MD 20814, USA (e-mail: jejackson@ usuhs.mil).

\section{REFERENCES}

1. Kroenke K, Mangelsdorff AD. Common symptoms in ambulatory care: incidence, evaluation, therapy, and outcome. Am J Med. 1989;86 (3):262-6.

2. Jackson JL, Passamonti M. The outcomes among patients presenting in primary care with a physical symptom at 5 years. J Gen Intern Med. 2005;20(11):1032-37.

3. Jewell D. I do not love thee $\mathrm{Mr}$ Fell...techniques for dealing with heartsink patients. BMJ. 1988;297:498-9.

4. Jackson JL, Kroenke K. Difficult patient encounters in the ambulatory clinic: clinical predictors and outcomes. Arch Intern Med. 1999;159: 1069-75.

5. Aaron LA, Buchwald D. A review of the evidence for overlap among unexplained clinical conditions. Ann Intern Med. 2001;134(9):868-81.

6. Petrou M, Harris RE, Foerster BR, et al. Proton MR spectroscopy in the evaluation of cerebral metabolism in patients with fibromyalgia: comparison with healthy controls and correlation with symptom severity. Am J Neuroradiol. 2008;29(5):913-8.

7. Diers M, Koeppe C, Yilmaz $\mathbf{P}$, et al. Pain ratings and somatosensory evoked responses to repetitive intramuscular and intracutaneous stimulation in fibromyalgia syndrome. J Clin Neurophysiol. 2008;25(3):153-60. 
8. Kroenke K, Spitzer RL, deGruy FV 3rd, et al. Multisomatoform disorder. An alternative to undifferentiated somatoform disorder for the somatizing patient in primary care. Arch Gen Psychiatry. 1997;54(4):352-8.

9. Jackson JL, Kroenke K. Prevalance, impact and prognosis of multisomatoform disorder in primary care: a 5-year follow-up study. Psychosom Med. 2008;70(4):430-4.

10. Jackson JL, Kroenke $\mathbf{K}$. The effect of unmet expectations among adults presenting with physical symptoms. Ann Intern Med. 2001;134:889-97.

11. Salmon P, Ring A, Humphries GM, Davies JC, Dowrick CF. Primary care consultations about medically unexplained symptoms: how do patients indicate what they want? $\mathrm{J}$ Gen Intern Med.

12. Peters S, Rogers A, Salmon P, et al. What do patients choose to tell their doctors? Qualitative analysis of potential barriers to reattributing medically unexplained symptoms. J Gen Intern Med.
13. Leiblum SR, Schnall E, Seehuus M, DeMaria A. To BATHE or not to BATHE: patient satisfaction with visits to their family physician. Fam Med. 2008;40(6):407-11.

14. Smith RC, Lyles JS, Gardiner JC, et al. Primary care clinicians treat patients with medically unexplained symptoms: a randomized controlled trial. J Gen Intern Med. 2006;21(7):671-7.

15. Tofferi JK, O'Malley PG, Jackson JL. Treatment of fibromyalgia with cyclobenzaprine: A metaanalysis. Arthritis Care Res. 2004;51 (1):9-13.

16. O'Malley PG, Jackson JL, Balden E, Tomkins G, Santoro J, Kroenke K. Antidepressant therapy for unexplained symptoms and symptom syndromes. J Fam Practice. 1999;48:980-99.

17. Kroenke $\mathbf{K}$. Efficacy of treatment for somatoform disorders: a review of randomized controlled trials. Psychosom Med. 2007;69(9):881-8. 